\title{
Changing Civil Servants' Awareness about Open Data Using a Collaborative Digital Game
}

\author{
Davide Di Staso \\ Delft University of Technology \\ The Netherlands \\ D.DiStaso@tudelft.nl \\ Joep Crompvoets \\ KU Leuven \\ Belgium \\ Joep.Crompvoets@kuleuven.be
}

\author{
Fernando Kleiman \\ Delft University of Technology \\ The Netherlands \\ F.Kleiman@tudelft.nl \\ Marijn Janssen \\ Delft University of Technology \\ The Netherlands \\ M.F.W.H.A.Janssen@tudelft.nl
}

\begin{abstract}
According to open government principles, public administration should make its data available to the public to create transparency, accountability, and to facilitate participation in public decision-making. Open-Data Policies (ODPs) were developed to encourage the opening of governmental data to the public, but require collaboration and awareness from all levels of government. Gaming has proved to be successful for creating awareness. Yet the pandemic hinders in-person gameplay, and there is a need for an online game that can be played remotely. This research uses an in-person game as the starting point to develop an online collaborative digital game. The feature and design choices of the digital game are described, along with its main differences when compared to the in-person one. The essential features include interaction and discussion about open data among players, the assignment of different roles, and points awarded when making the right decision to open, partially share, or close a dataset.
\end{abstract}

CCS Concepts: • Social and professional topics $\rightarrow$ Governmental regulations.

Keywords: serious games; gaming; collaborative learning; open data; open data policy; covid-19

ACM Reference Format:

Davide Di Staso, Fernando Kleiman, Joep Crompvoets, and Marijn Janssen. 2021. Changing Civil Servants' Awareness about Open Data Using a Collaborative Digital Game. In DG.O2021: The 22nd Annual International Conference on Digital Government Research (DG.O'21), June 9-11, 2021, Omaha, NE, USA. ACM, New York, NY, USA, 3 pages. https://doi.org/10.1145/3463677.3463684

Permission to make digital or hard copies of part or all of this work for personal or classroom use is granted without fee provided that copies are not made or distributed for profit or commercial advantage and that copies bear this notice and the full citation on the first page. Copyrights for thirdparty components of this work must be honored. For all other uses, contact the owner/author(s).

DG.O'21, fune 9-11, 2021, Omaha, NE, USA

(C) 2021 Copyright held by the owner/author(s).

ACM ISBN 978-1-4503-8492-6/21/06.

https://doi.org/10.1145/3463677.3463684

\section{Introduction}

Open data is part of the principles of open government, which entail that government should be open to interaction with other parties and act as an open system [3]. Open data policies are encouraging governments to release data to the public, but civil servants are often unaware of the data they collect, nor how it can be released [3]. The lack of awareness by public personnel can hinder opening of data by governments and has been targeted by different strategies. Simple communication (e.g. distributing leaflets) can work for some groups, but does not provide a deep understanding of the advantages of opening data, nor insights into how to release it. A possible solution is offered by experiential learning, which is the idea of simulating experiences in a safe environment [5]. Serious games can leverage the benefits of experiential learning in a risk-free environment and have been described as a promising tool to train civil servants [6]. Serious games can involve public personnel in open data decision-making and get them to experience data release, thereby creating awareness. Based on the concept of experiential learning, the idea is to influence attitudes through simulated experiences in a safe environment.

\section{Games on the topic of open data}

Previous games have been developed on the topic of open data: the Open Data Card game [2], aims at increasing data literacy, exploring how datasets can be used and combined, and create excitement about the potential applications of open data; Datopolis [1] is a board game developed at the Open Data Institute, its aim is to introduce players to open data without them "necessarily realising they're absorbing knowledge about open data" [1]; Datascape [9] is a board game which offers players an introduction to open data, it is designed for communities looking to leverage the power of open data in order identify and verify problems in their surroundings. Of all the games described, only Datopolis is available online through a sandbox simulating the environment of a tabletop game [7]. In order to specifically target 
civil servants' attitudes, Kleiman et al. [4] developed a collaborative role-playing game called Winning Data. When comparing surveys filled by players before and after the game sessions, it was possible to observe a change in awareness, with participants showing increased awareness about the open data they already provide, and a stronger future intention to provide more data. However, Winning Data had several limitations, such as (1) the requirement of in-person presence, which became prohibitive with the COVID-19 pandemic, and (2) the constant attention and effort of a trained facilitator, tasked with monitoring the game, distributing game materials, and keeping track of players' actions.

\section{Multiplayer games for collaborative learning}

A multiplayer digital game can still leverage the benefits of collaborative learning, such as positive interdependence and individual accountability [8], while at the same time removing the need for in-person presence and reducing the workload and training required from the facilitator. Wendel et al. [8] developed a serious game called Escape from Wilson Island, which can be played by groups of 2 to 4 players who need to collaborate together in order to escape from a deserted island. It was observed that players were able to solve common tasks as a team by communicating with each other [8]; also, the presence of a player assuming the role of team leader significantly improved the team's performance in collaborative tasks [8].

\section{Game description}

In order to leverage the benefits of serious gaming and collaborative learning in the context of open data, a new digital game called Data Belt (databelt.net) was developed. Data Belt is a digital co-operative video game for four players based on the analogue Winning Data by Kleiman et al. [4]. During the gameplay, which is guided by a facilitator, players need to be connected over audio or video conference in order to communicate. Like in its analogue counterpart, players are randomly assigned to four roles: citizen, civil servant, colleague and boss (figure 1). Players are tasked with processing data, an activity which requires all the specific skills of each different role (thereby requiring cooperation). After data processing, the citizen, civil servant and colleague need to propose a way to label each dataset (figure 2), which can be either completely open, partially shared, or completely closed. Any selection made by an individual player is immediately reflected on the other players' screens, who can revert it (in a similar fashion to a collaborative online document); therefore forcing the team to reach a consensus on one option. During preliminary test sessions, participants engaged in lively debates, discussing together the possible consequences of opening or closing a dataset. At the end of each round of the game, points are assigned to the entire

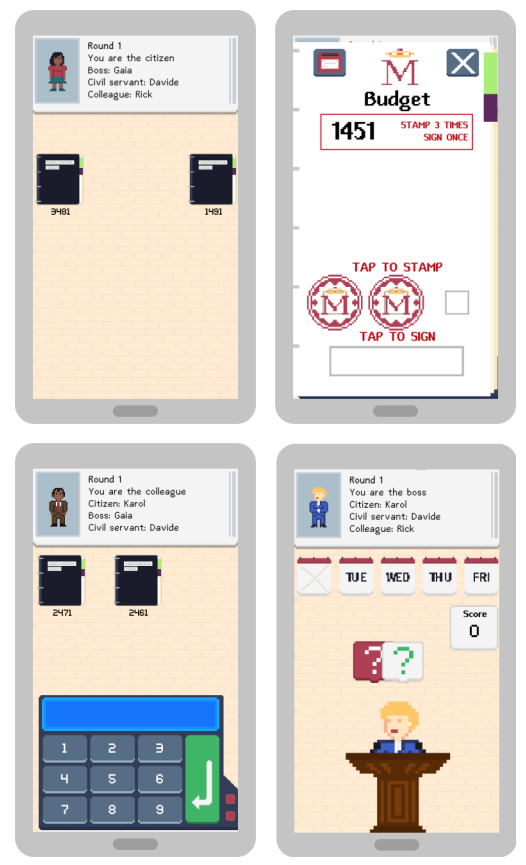

Figure 1. Four-player interaction in different roles (multiple screenshots).

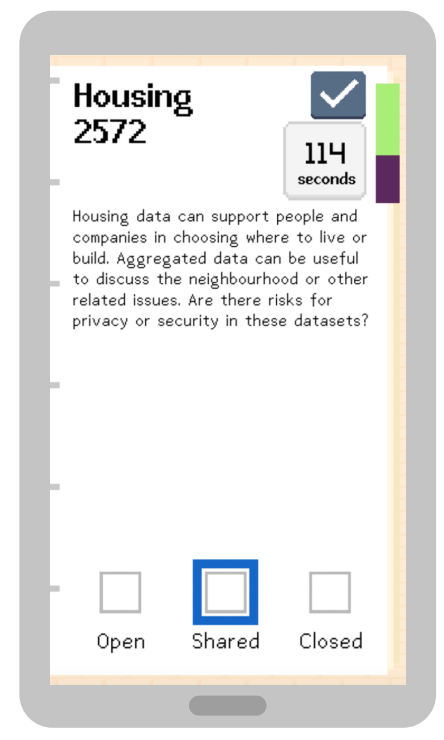

Figure 2. Dataset resulting from service delivery (screenshot). Dataset descriptions were adapted from Kleiman et al. [4].

team for each dataset correctly processed and labelled. The facilitator is able to see which datasets were labelled incorrectly and provides appropriate feedback so that the players can gain awareness about the benefits and risks of opening data. 


\section{Conclusions and future work}

This paper presented a prototype for a digital multiplayer game aimed at encouraging discussion and learning about open data among civil servants. The game requires collaboration without the need for in-person participation; it also offers an opportunity to experience decision-making about open data in a risk-free environment. Finally, the game provides civil servants with appropriate feedback when datasets are incorrectly opened or closed. Further iterations of the game will explore the possibility of completely replacing the facilitator with a tutorial mode to introduce new players to the game's mechanics and metaphor. Additionally, we plan to make the consequences of mistakes in the opening and closing of datasets more evident, given that in the current prototype they do not significantly impact the game's dynamics, thereby limiting the learning outcomes. Finally, we plan to implement new game modes allowing for groups smaller than 4 people to play together. The content of new developments and the assessment of the game's effects on civil servants will be the subject of future publications of this ongoing research.

\section{Acknowledgments}

For a detailed list of the open content assets used in the game see https://databelt.net/acknowledgements/

\section{References}

[1] Ellen Broad and Jeni Tennison. 2015. Datopolis Board Game. http: //datopolis.theodi.org/about/

[2] Jag Goraya. 2015. Open Data Card Game. https://github.com/Atomland/ open-data-card-game

[3] Marijn Janssen, Yannis Charalabidis, and Anneke Zuiderwijk. 2012. Benefits, Adoption Barriers and Myths of Open Data and Open Government. Information Systems Management 29, 4 (Sept. 2012), 258-268. https://doi.org/10.1080/10580530.2012.716740

[4] Fernando Kleiman, Marijn Janssen, and Sebastiaan Meijer. 2019. Evaluation of a Pilot Game to Change Civil Servants' Willingness towards Open Data Policy Making. In Simulation and Gaming: Through Times and across Disciplines. Kozminski University, Warsaw, 286-297.

[5] David A. Kolb. 2000. The Process of Experiential Learning. In Strategic Learning in a Knowledge Economy. Elsevier, 313-331. https://doi.org/ 10.1016/B978-0-7506-7223-8.50017-4

[6] Karol Olejniczak, Tomasz Kupiec, and Igor Widawski. 2016. Knowledge Brokers in Action: A Game-Based Approach for Strengthening Evidence-Based Policies. In Simulation and Gaming in the Network Society, Toshiyuki Kaneda, Hidehiko Kanegae, Yusuke Toyoda, and Paola Rizzi (Eds.). Springer Singapore, Singapore, 427-441.

[7] David Tarrant. 2020. Moving Datopolis Online. http://datopolis.theodi. org/blog/2020-04-23-lockdown

[8] Viktor Wendel, Michael Gutjahr, Stefan Göbel, and Ralf Steinmetz. 2013. Designing Collaborative Multiplayer Serious Games: Escape from Wilson Island-A Multiplayer 3D Serious Game for Collaborative Learning in Teams. Education and Information Technologies 18, 2 (June 2013), 287-308. https://doi.org/10.1007/s10639-012-9244-6

[9] Annika Wolff, Matthew Barker, and Marian Petre. 2017. Creating a Datascape: A Game to Support Communities in Using Open Data. In 8th International Conference on Communities and Technologies. ACM, New York, NY, USA, 135-138. 